\title{
Erratum to: An industry-level blu-ray watermarking framework
}

\author{
Jan De Cock • Heinz Hofbauer • Thomas Stütz • \\ Andreas Uhl • Andreas Unterweger
}

Published online: 15 March 2015

(C) Springer Science+Business Media New York 2015

\section{Erratum to: Multimed Tools Appl \\ DOI 10.1007/s11042-014-2042-y}

During the publication process, a typographical error was introduced. The correct equation appears below:

$$
\operatorname{pfp}(C)=\sum_{k=k c}^{n}\left(\begin{array}{l}
n \\
k
\end{array}\right) p^{k} q^{(n-k)}=\sum_{k=k c}^{n}\left(\begin{array}{l}
n \\
k
\end{array}\right)\left(\frac{1}{2}\right)^{n}=\frac{1}{2^{n}} \sum_{k=k c}^{n}\left(\begin{array}{l}
n \\
k
\end{array}\right)
$$

The online version of the original article can be found at http://dx.doi.org/10.1007/s11042-014-2042-y.

J. De Cock

Ghent University-iMinds, Gaston Crommenlaan 8 bus 201, B-9050 Ledeberg-Ghent, Belgium e-mail: jan.decock@ugent.be

H. Hofbauer $(\square) \cdot$ A. Uhl $\cdot$ A. Unterweger

University of Salzburg, Jakob Haringer Str. 2, 5020 Salzburg, Austria

e-mail: hhofbaue@cosy.sbg.ac.at

A. Uhl

e-mail: uhl@cosy.sbg.ac.at

A. Unterweger

e-mail: aunterweg@cosy.sbg.ac.at

T. Stütz

FH Salzburg, Urstein Süd 1, 5412 Puch bei Hallein, Austria

e-mail: thomas.stuetz@fh-salzburg.ac.at 